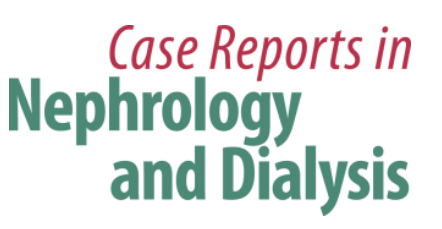

Case Rep Nephrol Dial 2018;8:35-44

DOI: $10.1159 / 000487262$

Published online: March 2, 2018

(C) 2018 The Author(s)

Published by S. Karger AG, Basel

www.karger.com/cnd

This article is licensed under the Creative Commons Attribution-NonCommercial 4.0 International License (CC BY-NC) (http://www.karger.com/Services/OpenAccessLicense). Usage and distribution for commercial purposes requires written permission.

\title{
The Convergence of Vasculopathy and Vasculitis: Computer Mapping Analysis of 2 Renal Biopsies in a Patient with both Systemic Sclerosis and ANCA-Related Vasculitis
}

\author{
Jia $\mathrm{Xu}^{\mathrm{a}} \quad$ Milan Rosen $^{\mathrm{a}} \quad$ Christina I. Luffman ${ }^{\mathrm{a}}$ Charles Law $^{\mathrm{c}}$ \\ Anita Laloo $^{\mathrm{b}}$ Seymour Rosen ${ }^{\mathrm{a}}$ Beverly E. Faulkner-Jones ${ }^{\mathrm{a}}$ \\ ${ }^{a}$ Department of Pathology, Beth Israel Deaconess Medical Center, Harvard Medical \\ School, Boston, MA, USA; ${ }^{\circ}$ Division of Rheumatology, Department of Medicine, Beth Israel \\ Deaconess Medical Center, Harvard Medical School, Boston, MA, USA; ${ }^{C}$ Kitware Inc., \\ Clifton Park, NY, USA
}

\section{Keywords}

Vasculopathy · Vasculitis · Computer mapping · Renal biopsy · Systemic sclerosis . Antineutrophil cytoplasmic antibodies-related vasculitis

\begin{abstract}
Scleroderma vasculopathy and ANCA (antineutrophil cytoplasmic antibodies)-associated glomerulonephritis have rarely been reported to occur simultaneously in one patient. Herein, we report a patient who presented with a classic constellation of clinical and laboratory findings of systemic scleroderma and was subsequently found to be positive for p-ANCA. Two renal biopsies, performed 5 months apart, demonstrated typical changes of the two entities in both acute and "healed" phases, which were analyzed by computer mapping techniques. The two renal biopsies were serially sectioned and stained routinely, and with CD31 and CD34 as endothelial markers. The slides were digitized, aligned and analyzed. Each glomerular tuft was sequentially studied in terms of total area $\left(\mu \mathrm{m}^{2}\right)$ and each biopsy was individually profiled. All arterial vessels were sequentially studied with whole vessel and luminal areas delineated and ratios calculated. The initial biopsy contained 32 glomeruli almost all with
\end{abstract}




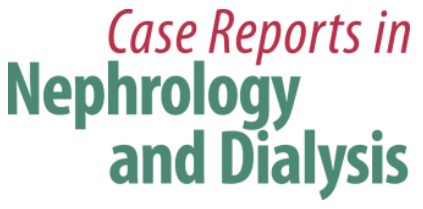

Case Rep Nephrol Dial 2018;8:35-44

DOI: $10.1159 / 000487262$

(C) 2018 The Author(s). Published by S. Karger AG, Basel www.karger.com/cnd

Xu et al.: Convergence of Vasculopathy and Vasculitis: Computer Mapping Analysis of 2 Renal Biopsies in a Patient with both Systemic Sclerosis and ANCA-Related Vasculitis

extensive fibrinoid necrosis and destruction of the capillary network. The arterial vessels (interlobular and arcuate) showed intimal edema with luminal occlusion. CD31/CD34 stains showed variable endothelial intactness but demonstrated the luminal size shifts. The second biopsy had 37 glomeruli that were either segmentally or globally sclerotic with no active changes. The vessels were now normally patent. Each glomerular tuft and arterial vessel in both biopsies was analyzed as a serial section histogram documenting these changes. These studies depict the rare occurrence of two entities together, the scleroderma kidney vasculopathy and the glomerulonephritis of ANCA-associated vasculitis syndrome both in an acute and healing phase, profiled by computer mapping techniques.

(C) 2018 The Author(s)

Published by S. Karger AG, Basel

\section{Introduction}

There are 2 major categories of so-called "immune-related" vascular injury, namely vasculopathy and vasculitis, which are separated largely but not completely by the degree and quality of the inflammatory component. Vasculitides are usually characterized by active inflammation and include antineutrophil cytoplasmic antibodies (ANCA)-associated and cutaneous leukocytoclastic vasculitides of various etiologies (e.g., Henoch-Schönlein purpura, drug-related vasculitis) but can be a more chronic process, such as giant cell arteritis. With repair, inflammation decreases and fibrosis ensues. In entities characterized by a vasculopathy, the process seems that of a primary endothelial injury. Such diseases (e.g., thrombotic microangiopathy, scleroderma, and dermatomyositis) are characterized by an apparent endothelial mesenchymal transition that often results in lumenal occlusion [1-3]. There is, as well, overt capillary loss that is defined by nail bed studies in scleroderma and dermatomyositis [4]. Muscle and skin biopsies have demonstrated a decrease in the number of capillaries as well as capillary loop dilatation [5]. This diminishment of the microvasculature ultimately occurs in multiple organs. In both categories, there are varying degrees of coagulationfibrinolytic system involvement.

The patient presented herein had clinically both an ANCA-associated vasculitic syndrome and scleroderma. These entities have been demonstrated to rarely occur together [6]. This report is the first to describe their characteristic morphological findings seen simultaneously in a single renal biopsy suggesting closely related pathogenetic mechanisms. Furthermore, these biopsies were analyzed by digital profiling, which quantified both the active and reparative processes.

\section{Materials and Methods}

A 44-year-old man was admitted after he was referred by his primary care physician for weight loss and a decreased appetite. The patient stated that he felt well until approximately 2 months prior to admission when he developed a flu-like illness. He subsequently had a 40pound weight loss, extreme weakness and fatigue. His review of systems was negative for joint pain/swelling, rash, oral ulcers, nausea, abdominal pain, diarrhea, chest pain, dyspnea, dysphagia, or odynophagia.

His physical exam was notable for sclerodactyly with digital ulcers and pitting on his bilateral hand exam with skin tightening on his fingers and the dorsal aspect of his hands extending to his distal forearms. There was limited flexion and extension of his digits at the 


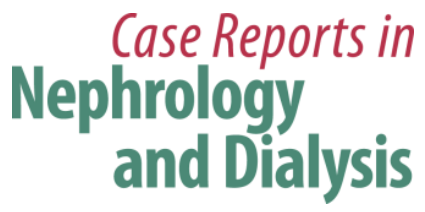

distal interphalangeal and proximal interphalangeal joints with scattered areas of skin hypopigmentation on the dorsal aspects of his fingers. Nail changes with onycholysis as well as a decreased oral aperture were prominent findings. Laboratory test from his initial office visit showed hemoglobin $9.9 \mathrm{~g} / \mathrm{dL}$, hematocrit $31.6 \%$, normal platelet count, and a creatinine of $1.3 \mathrm{mg} / \mathrm{dL}$.

At the emergency department 4 weeks later, his vital signs were notable for a temperature of $99.8^{\circ} \mathrm{F}$, mild tachycardia to 102 , and blood pressure of $127 / 75 \mathrm{~mm} \mathrm{Hg}$ with oxygen saturation of $100 \%$ on room air. He also had an elevated white blood cell count to $23.9 \mathrm{~K} / \mu \mathrm{L}$ with $90 \%$ neutrophils, severe anemia with hemoglobin $4.2 \mathrm{~g} / \mathrm{dL}$, and hematocrit $13.6 \%$ with a platelet count of $473 \mathrm{~K} / \mu \mathrm{L}$. His blood urea nitrogen and creatinine were elevated to 135 $\mathrm{mg} / \mathrm{dL}$ and $8.8 \mathrm{mg} / \mathrm{dL}$, respectively, with hypoalbuminemia to $1.7 \mathrm{~g} / \mathrm{dL}$ and an elevated Creactive protein to $187 \mathrm{mg} / \mathrm{L}$. Initial urinalysis showed $30 \mathrm{mg} / \mathrm{dL}$ protein, 30 red blood cells per high-power field, and 6 white blood cells per high-power field with a urine protein to creatinine ratio of 0.9 .

Prior to his admission, a computed tomography scan was notable for bilateral scattered subpleural opacities, extensive bilateral chronic aspiration with dilated, patulous esophagus suggestive of achalasia, and bilateral axillary lymphadenopathy. Based on findings of the nail changes and skin thickening over both hands, systemic sclerosis (SSc) was diagnosed based on the 2013 American College of Rheumatology criteria. The patient received 2 units of packed red blood cells and intravenous methylprednisolone followed by a renal biopsy. On biopsy, there was an extensive necrotizing crescentic glomerulonephritis consistent with the ANCA-associated vasculitic syndrome combined with findings of vascular changes seen in the scleroderma kidney (see Results section). Subsequent labs showed positive antinuclear antibodies at a titer of 1:640 with a speckled pattern, positive rheumatoid factor with negative results for anti-scl-70, anti-Ro and La, anti-Sm, anti-ribonucleoprotein, RNA polymerase III, anti-cyclic citrullinated peptide, and anti-glomerular basement membrane antibodies. ANCA was strongly positive with pANCA titer $>1: 640$ and a positive anti-myeloperoxidase (MPO) antibody.

He was managed with 10 cycles of plasmapheresis and pulse dose steroids followed by cyclophosphamide at $1.5-2 \mathrm{mg} / \mathrm{kg}$ daily to which his renal function responded well with an improvement in his creatinine $(3.7 \mathrm{mg} / \mathrm{dL})$. He was discharged after 6 weeks.

At clinic visit 5 months later, it was noted that he had worsened renal function, persistently positive ANCA titer, increase in creatinine $(4.5 \mathrm{mg} / \mathrm{dL})$, elevated erythrocyte sedimentation rate $(58 \mathrm{~mm} / \mathrm{h})$, and leukopenia. Repeat renal biopsy was performed which showed severe chronic disease with marked global and segmental glomerulosclerosis, fibrous crescents, and severe interstitial fibrosis and tubular atrophy (see Results section). Cyclophosphamide was subsequently discontinued, and the patient was started on azathioprine for extrarenal manifestations. His clinical symptoms and renal function have been stable since with the most recent follow-up available 22 months after hospital discharge.

\section{Results}

\section{Pathologic Findings}

Both biopsies were processed using the usual protocol (H\&E, PAS, Masson trichrome, and silver methenamine), immunofluorescence, and electron microscopy, but additionally endothelial markers (CD31, CD34) were performed as well. 


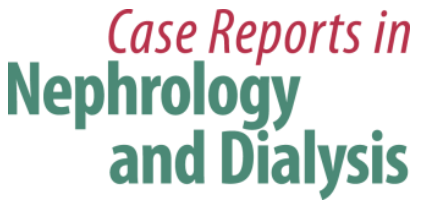

Case Rep Nephrol Dial 2018;8:35-44

DOI: $10.1159 / 000487262$

(C) 2018 The Author(s). Published by S. Karger AG, Basel www.karger.com/cnd

Xu et al.: Convergence of Vasculopathy and Vasculitis: Computer Mapping Analysis of 2 Renal Biopsies in a Patient with both Systemic Sclerosis and ANCA-Related Vasculitis

The initial renal biopsy (biopsy 1) at the time of diagnosis in this patient included approximately 32 glomeruli almost all of which showed prominent fibrinoid change associated with epithelial crescent formation and focal destruction of Bowman's capsule (Fig. 1). Minimal endocapillary proliferation was seen. There was fairly diffuse interstitial inflammatory infiltrate composed of plasma cells, lymphocytes, and neutrophils accompanied by mild fibrosis. The arterioles and small interlobular arteries showed focal edematous intimal thickening, and 1 arcuate artery was nearly occluded by a combination of fibrosis and edema. These changes were seen very clearly in the CD34/CD31 preparations which defined the destruction of the glomerular capillary network and the arterial narrowing with associated endothelial injury.

Immunofluorescence studies showed no significant immunoreactants, but intense fibrin staining was noted both within the glomerular tuft and the urinary space. Electron microscopic studies of the glomeruli showed extensive capillary wall destruction, fibrin deposition, and epithelial proliferation, as expected. In a large artery, the media was seen to be relatively intact, but the intima was expanded by myofibroblastic proliferation with areas of diminished matrix likely due to edema. A diagnosis of extensively necrotizing crescentic glomerulonephritis consistent with the ANCA-associated vasculitic syndromes was made with a comment that no literal vasculitis was identified. However, the arteries demonstrated edema and intimal fibroplasia which were more characteristic of SSc.

A repeat renal biopsy performed 5 months after initiation of treatment (biopsy 2) showed predominantly chronic changes, including glomerular sclerosis of most glomeruli and fibrous crescents. Marked interstitial fibrosis and tubular atrophy were noted accompanied by focal chronic inflammation. The arterioles/interlobular arteries also demonstrated mural fibrosis but were patent. These changes in this biopsy were essentially chronic and related to the previous necrotizing crescentic glomerulonephritis.

\section{Computer Data Analysis}

Serial sections of each biopsy were digitized and uploaded onto a digital pathology viewing system (Slide Atlas, Kitware, NY, USA). Manual segmentation was done of the glomeruli and arteries (external media and lumen delineated). The lumen/total arterial area was calculated as a percentage.

The glomerular tufts were delineated by utilizing a Bowman's capsule as a demarcating point. In biopsy 1, the area included the glomerular tufts and the associated crescent.

The presence of fragmentation of Bowman's capsular basement membrane made this measurement approximate. Each glomerulus was individually outlined and the area evaluated to create sequential values from aligned slides. Then, histograms were made with the vertical axis representing glomerular area $\left(\mu \mathrm{m}^{2}\right)$ and the horizontal axis representing individual serial section number (Fig. 2). Another way to present the data more simply was as a bar graph representing maximal size from each glomerular histogram. Glomerular diameter (approximately $220 \mu \mathrm{m}=$ normal value) is a value which would determine the completeness of histogram analysis [7].

The total amount of tissue examined is an important consideration. There are approximately 4 sections per slide ( $3 \mu \mathrm{m}$ thickness); placing the paraffin ribbon on the slide results in the loss of the 2 lateral sections per each slide. The total amount of tissue cut can be estimated by multiplying the number of profiles by $3 \mu \mathrm{m}$. Biopsy 1 included 85 profiles on 23 slides (total tissue thickness cut $=262 \mu \mathrm{m}$ ), and biopsy 2 included 82 profiles on 20 slides (total tissue thickness cut $=236 \mu \mathrm{m}$ ). 


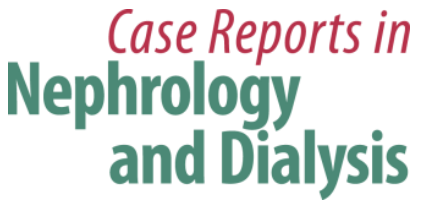

Case Rep Nephrol Dial 2018;8:35-44

DOI: $10.1159 / 000487262$

(C) 2018 The Author(s). Published by S. Karger AG, Basel www.karger.com/cnd

Xu et al.: Convergence of Vasculopathy and Vasculitis: Computer Mapping Analysis of 2 Renal Biopsies in a Patient with both Systemic Sclerosis and ANCA-Related Vasculitis

Overall, biopsy 1 showed marked enlargement of the glomeruli. These large glomeruli would be partially represented by histogram given that the diameters of many of the larger glomeruli are larger than the total thickness sectioned. The smaller values in biopsy 1 were related to a single glomerular tuft which was normal. On the other hand, the highest values in biopsy 2 are likely related to chronic hypertrophic changes (enlarged normal glomeruli) secondary to diminished nephron mass, the smallest global sclerosis. Thus, histograms in biopsy 2 would in effect have a more complete representation of most of its glomeruli.

The segmentation of arterial vessels presented problems. The number of arteries represented with multiple profiles in these biopsies was few, and orientation became a problem. In Figure 3 (biopsy 1), a single vessel retained its cross-sectional profile in multiple levels, showing a dramatically diminished lumen. The problem of orientation is illustrated in biopsy 2 , which shows a vessel with numerous profiles shifting from a full cross-section to an oblique confirmation.

\section{Discussion}

SSc is a multisystem connective tissue disorder characterized by vasculopathy, fibrosis, and autoimmunity affecting the skin and various internal organs, including the lung, heart, kidney, and gastrointestinal tract. The precise etiology of SSc is unknown. However, evidence indicates that vascular damage is a primary event in the pathogenesis of SSc [8]. The generally accepted pathogenesis is that initial vascular injury due to autoimmunity and/or environmental factors causes structural and functional abnormalities of vasculature which eventually result in constitutive activation of fibroblasts in various organs. The vasculopathy in this entity consists of destruction of small vessels (loss of capillaries) and an obliterative vasculopathy of arterioles and arteries characterized by fibroproliferative changes.

Impaired function of vasculature in SSc includes the altered expression of cell adhesion molecules predominantly inducing Th2 and Th17 cell infiltration and endothelial dysfunction primarily due to the low availability of nitric oxide. Several vascular biomarkers, including VEGF, PDGF- $\beta$, endothelin-1, ICAM-1, VCAM, and E-selectin, have been shown to have altered levels of expression in association with the dysregulation of angiogenesis and vasculogenesis in a variety of organs [9]. Activated endothelial-to-mesenchymal transition leads to occlusive vascular change and tissue fibrosis in concert with an impaired coagulation/fibrinolysis system that promotes the formation of intravascular fibrin deposits. A growing body of evidence suggests that deficiency of the transcription factor Friend, leukemia integration factor-1 (Fli1), may have a significant role in the development of both skin fibrosis and micro-vessel injury in SSc [10].

Vascular dysfunction in SSc contributes significantly to the mortality and morbidity of this condition. Renal disease in SSc usually manifests as a precipitous event with accelerated hypertension, acute renal failure, and mild proteinuria but can occur with normal blood pressure as in this patient. The primary mechanism of scleroderma renal crisis is thought to be vasospasm leading to proliferative vasculopathy of the arcuate and interlobular arteries with fibrin thrombi and areas of fibrinoid necrosis seen acutely. Glomeruli are affected secondarily. Later changes result in fibrosis with accumulation of extracellular matrix in the glomerular and tubulointerstitial compartments, mucoid intimal thickening, and concentric onion-skin hypertrophy of the interlobular arteries [11].

In about $20-30 \%$ of patients with SSc, nonspecific ANCA reactivity can be found with anti-MPO antibody being more common than anti-proteinase 3 antibody. A small subset of 


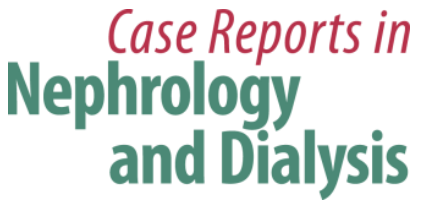

Case Rep Nephrol Dial 2018;8:35-44

DOI: $10.1159 / 000487262$

(C) 2018 The Author(s). Published by S. Karger AG, Basel www.karger.com/cnd

Xu et al.: Convergence of Vasculopathy and Vasculitis: Computer Mapping Analysis of 2 Renal Biopsies in a Patient with both Systemic Sclerosis and ANCA-Related Vasculitis

these patients also presents with symptoms of ANCA-associated systemic vasculitis. The combination of systemic vasculitis and SSc is unusual but has been reported in 2 retrospective case reviews $[12,13]$. ANCA-associated vasculitis in the kidney has glomerular changes that belong to a class of findings termed "crescentic glomerulonephritis." The pathology is mediated by injury of glomerular capillaries and endothelial cells. The rupture of the glomerular capillary walls allows fibrin, plasma proteins, inflammatory mediators, and leukocytes to enter Bowman's space, where they induce the proliferation of epithelial cells and infiltration of macrophages to produce cellular crescents. Injury of the glomerular capillary endothelium is obviously the critical factor in this injury sequence.

In this study, we have characterized the vascular injury both in vessels and glomeruli by using endothelial markers (CD31 and CD34). Indeed, previous studies have demonstrated that immunohistochemical staining with CD34 is a useful technique for assessing the integrity of glomerular tuft capillaries [14]. Using this technique, the glomerular endothelium is seen to be extensively fragmented, and the endothelium of vessels focally showed such fragmentation as well. Circulating microparticles derived from both endothelium and platelets have been documented in both scleroderma and ANCA-associated glomerulonephritis [15].

These biopsies with 2 morphologically distinct processes occurring simultaneously in separate compartments (glomeruli and vessels) provided a unique opportunity for computerized analysis. In essence, changes typical of ANCA-associated glomerulonephritis (necrotizing crescentic glomerulonephritis) and alterations typical of SSc renal crises (arterial intimal edema and fibroplasia) were simultaneously present in the first biopsy and reparative changes in the second. The serial sections allowed a different analytic approach, i.e., both glomeruli and vessels were followed sequentially in each biopsy, providing an ability to create histogram profiles of these structures. The glomerular tufts in the first biopsy were markedly enlarged. The healing phase showed a reduction in size and structural irregularity; interestingly, a few glomeruli were larger (evidently a hypertrophic response of intact nephrons). Analysis of the vasculature was more challenging because of the limited numbers available and other factors that were not anticipated. The extreme narrowing was defined and quantitated, and this was obvious in biopsy 1 . The healed vessels in biopsy 2 were surprisingly normal, so that the walls were thinner and less rigid, the lumen being smaller because of collapse.

Study by this computer mapping technique allowed a much more quantitative approach to the analysis of these 2 renal biopsies. Both an active phase and the healing process were evaluated in this way and provided our first approach to this type of renal biopsy analysis. Each glomerular tuft and arterial vessel was analyzed in serial sections, and the histograms provided a completely different perspective of the glomerular changes. The manual segmentation to do this study was very labor intensive, but we believe it provided proof of concept that computer mapping can offer significant information in the study of renal biopsies. We are now working actively on methodology for automated segmentation so this can be much more easily applied.

\section{Conclusion}

We report a case demonstrating findings of ANCA-associated vasculitis and scleroderma renal crisis in both active and healing phases. CD34 and CD31 were useful markers in the evaluation of glomerular endothelial integrity as well as in the diagnosis of vasculitis and 
vasculopathy. Computerized analysis (glomerular and vascular profiling) in sequential sections, as a novel method, provides another dimension for more objective characterization of these glomerular and vascular changes.

\section{Acknowledgment}

Steve Turney, Center for Brain Science, Harvard University, Cambridge, MA, and Brian Helba, Kitware, provided database support.

\section{Statement of Ethics}

The authors have no ethical conflicts to disclose.

\section{Disclosure Statement}

The authors declare no conflicts of interest.

\section{Funding Sources}

Digital imaging technique was developed with support from NIBIB SBIR Grant R44EB013518-02A, NIH R44MH08808 8 04, and the Harvard Catalyst Advanced Microscopy Pilot Grant.

\section{References}

Asano Y, Sato S: Vasculopathy in scleroderma. Semin Immunopathol 2015;37:489-500. Gabrielli A, Avvedimento EV, Krieg T: Scleroderma. N Engl J Med 2009;360:1989-2003. Vardeh H, Magro CM, Brown L, et al: Gastrointestinal pathology associated with dermatomyositis: presentation of 3 cases and a general review. Am J Surg Pathol Rev Rep 2016;21:293-300. Spencer-Green G, Schlesinger M, Bove KE, et al: Nailfold capillary abnormalities in childhood rheumatic diseases. J Pediatr 1983;102:341-346.

Rabquer BJ, Koch AE: Angiogenesis and vasculopathy in systemic sclerosis: evolving concepts. Curr Rheumatol Rep 2012;14:56-63.

Arnaud L, Huart A, Plaisier E, et al: ANCA-related crescentic glomerulonephritis in systemic sclerosis: revisiting the "normotensive scleroderma renal crisis." Clin Nephrol 2007;68:165-170.

humans. Ultrasound Med Biol 1996;22:987-997.

Manetti M, Guiducci S, Ibba-Manneschi L, et al: Mechanisms in the loss of capillaries in systemic sclerosis: angiogenesis versus vasculogenesis. J Cell Mol Med 2010;14:1241-1254.

Chora I, Guiducci S, Manetti M, et al: Vascular biomarkers and correlation with peripheral vasculopathy in systemic sclerosis. Autoimmun Rev 2015;14:314-322.

Noda S, Asano Y, Nishimura S, et al: Simultaneous downregulation of KLF5 and Fli1 is a key feature underlying systemic sclerosis. Nat Commun 2014;5:5797.

Woodworth TG, Suliman YA, Furst DE, et al: Scleroderma renal crisis and renal involvement in systemic sclerosis. Nat Rev Nephrol 2016;12:678-691.

Quéméneur T, Mouthon L, Cacoub P, et al: Systemic vasculitis during the course of systemic sclerosis: report of 12 cases and review of the literature. Medicine (Baltimore) 2013;92:1-9.

Derrett-Smith EC, Nihtyanova SI, Harvey J, et al: Revisiting ANCA-associated vasculitis in systemic sclerosis: clinical, serological and immunogenetic factors. Rheumatol Oxf Engl 2013;52:1824-1831. 


\section{Case Reports in Nephrology and Dialysis}

\begin{tabular}{l|l}
\hline Case Rep Nephrol Dial 2018;8:35-44 \\
\hline DOI: 10.1159/000487262 & $\begin{array}{l}\text { C 2018 The Author(s). Published by S. Karger AG, Basel } \\
\text { www.karger.com/cnd }\end{array}$ \\
\hline
\end{tabular}

$\mathrm{Xu}$ et al: Convergence of Vasculopathy and Vasculitis: Computer Mapping Analysis of 2 Renal Biopsies in a Patient with both Systemic Sclerosis and ANCA-Related Vasculitis

14 Fujita E, Nagahama K, Shimizu A, et al: Glomerular capillary and endothelial cell injury is associated with the formation of necrotizing and crescentic lesions in crescentic glomerulonephritis. J Nippon Med Sch 2015;82:27-35.

15 Erdbrügger U, Le TH: Extracellular vesicles in renal diseases: more than novel biomarkers? J Am Soc Nephrol 2016;27:12-26.

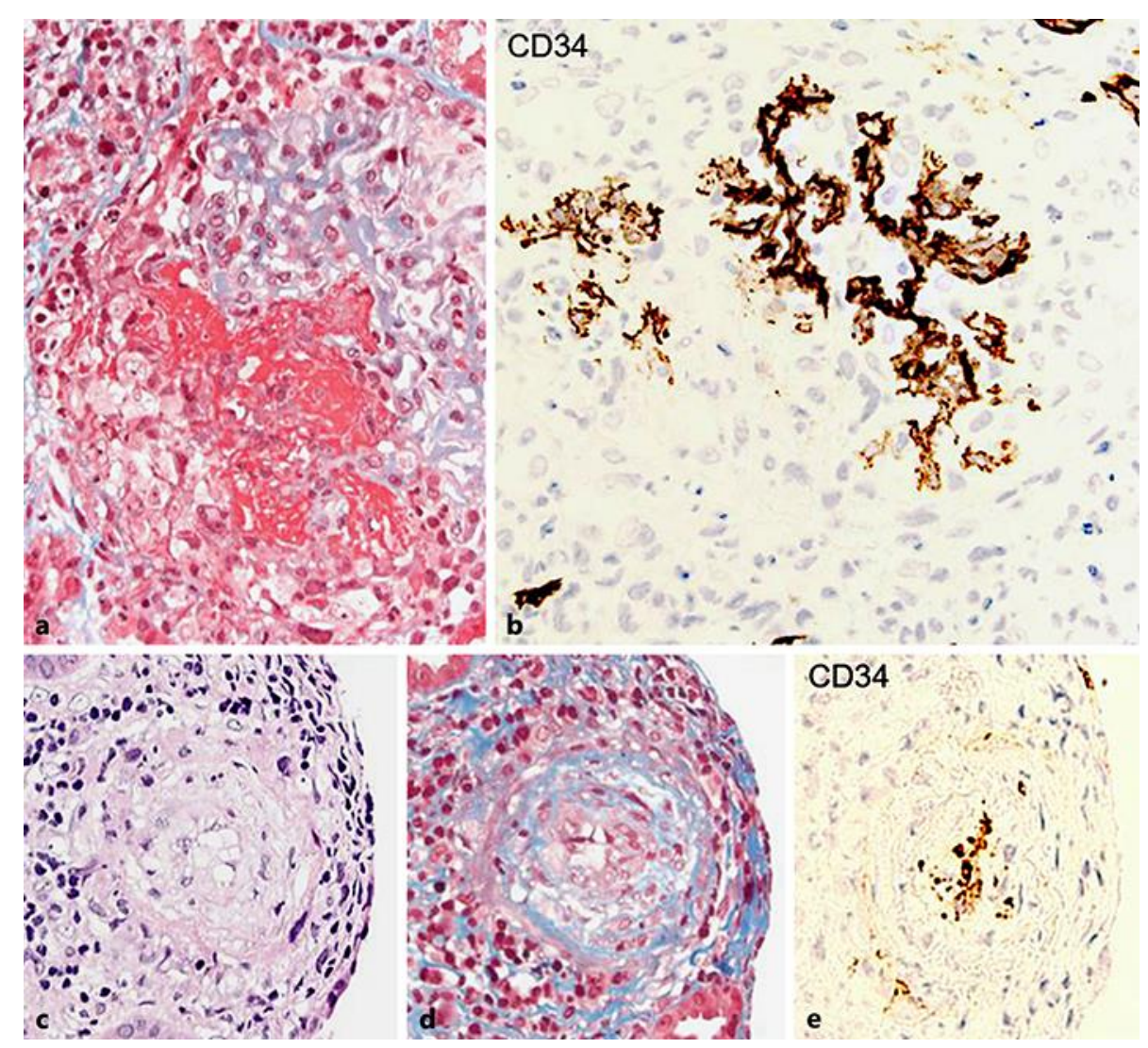

Fig. 1. Glomerular and vascular changes in biopsy (H\&E). a The extensive glomerular destruction and fibrin deposition are well seen in the Masson trichrome preparation. Endothelial marker (CD34) stains (b) delineate the disruption of the glomerular capillary network with endothelial loss/fragmentation. The arterial intima edema is well seen (H\&E, c; Masson trichrome, d), and the CD34 stain (e) demarcates the narrowed arterial lumen and shows fragmented endothelium. 


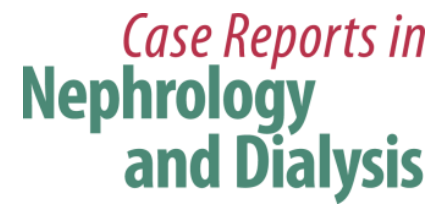

Case Rep Nephrol Dial 2018;8:35-44

(C) 2018 The Author(s). Published by S. Karger AG, Basel www.karger.com/cnd

Xu et al: Convergence of Vasculopathy and Vasculitis: Computer Mapping Analysis of 2 Renal Biopsies in a Patient with both Systemic Sclerosis and ANCA-Related Vasculitis
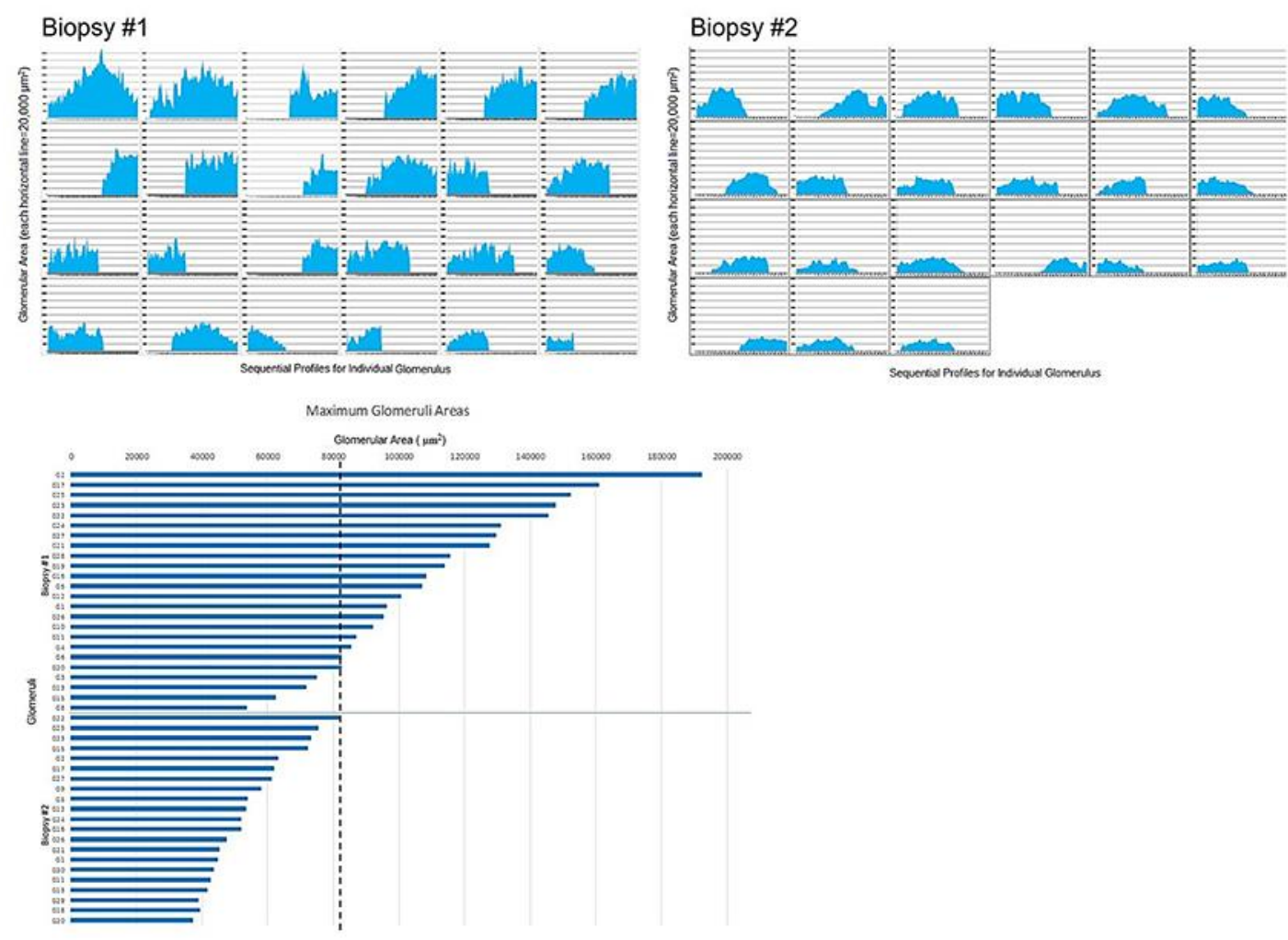

Fig. 2. Top: composite of sequential glomerular area histograms from each biopsy (each horizontal line on the vertical axis $=20,000 \mu \mathrm{m}^{2}$ ). In biopsy 1 , profiles are seen which demonstrate the very large glomerular tufts present and the incomplete histograms because of size constraints. On the other hand, histograms in biopsy 2 illustrate smaller glomeruli with more complete histograms. Bottom: bar graph representing maximal size from glomerular histograms. Biopsy 1 (upper) shows the overall marked enlargement of the glomeruli; smaller values in biopsy 1 were related to a single glomerular tuft, which was normal, and incomplete representation of these large glomeruli. The highest values in biopsy 2 (lower) are likely related to hypertrophic changes, the smallest global sclerosis. 


\section{Case Reports in Nephrology and Dialysis}
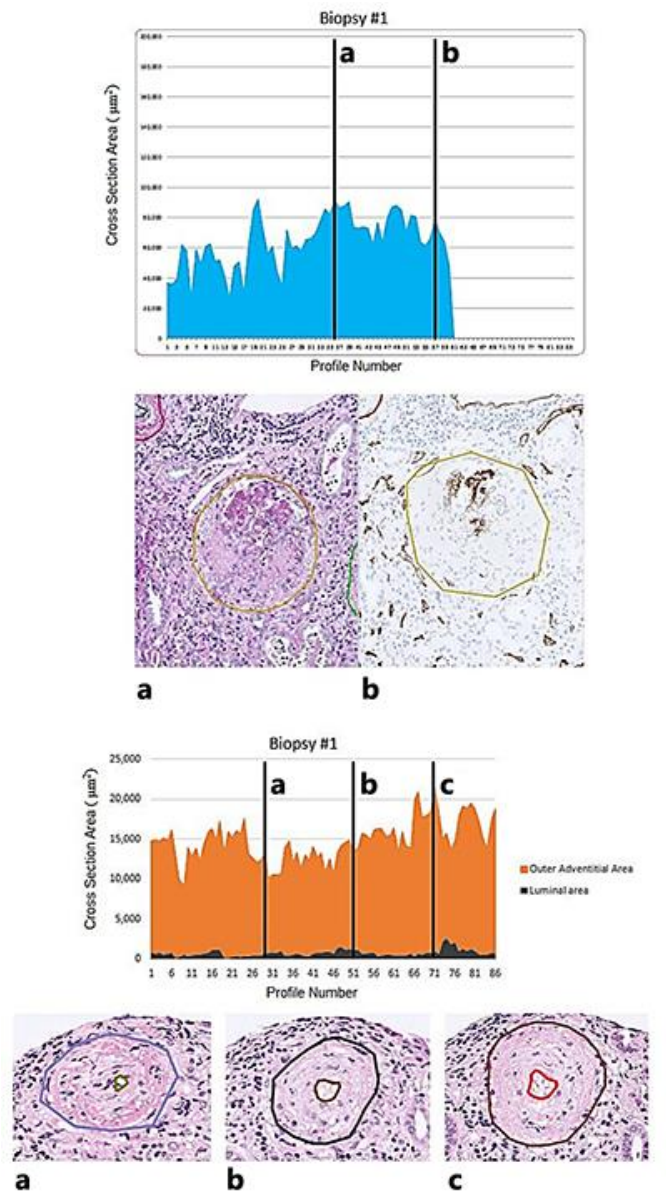

Xu et al: Convergence of Vasculopathy and Vasculitis: Computer Mapping Analysis of 2 Renal Biopsies in a Patient with both Systemic Sclerosis and ANCA-Related Vasculitis

Fig. 3. Top: individual histograms are seen with segmented and profiled glomeruli (PAS, CD31) and vessels (H\&E); bars indicate source of the image from profile (biopsy 1, left; biopsy 2, right). Bottom: the segmentation of arterial vessels presented problems (few numbers and orientation). In biopsy 1 (left), a single vessel retained its cross-sectional profile in multiple levels, showing a dramatically diminished lumen. In biopsy 2 (right), a vessel is shown with numerous profiles shifting from a full cross-section to an oblique conformation. 